\title{
A VISITA DO ROBERT PARK AO BRASIL, O "HOMEM MARGINAL" E A BAHIA COMO LABORATÓRIO
}

\author{
Licia do Prado Valladares*
}

\begin{abstract}
Este artigo insere-se nas preocupações teóricas da autora sobre a recepção da "Escola de Chicago", no Brasil. Analisa a passagem de Robert Park ao final dos anos 30 na Bahia; suas motivações e os efeitos para as ciências sociais internacionais. Essa viagem é pouco conhecida e o artigo traz uma pequena colaboração para a história das ciências sociais, no Brasil e na Bahia. Baseada em dados originais de pesquisa realizada no Brasil e nos Estados Unidos, o autora considera a importância da visita de Park [e de Pierson] à Bahia. Retoma as noções clássicas de Homem Marginal, desenvolvida por Park e de melting pot, usada por Park e discípulos, ao se referirem à convivência, em Chicago, de comunidades com nacionalidades diferentes, que não se misturavam. O caso baiano, de miscigenação, intrigou Park, que acabou transformando a Bahia num "laboratório social", suscitando a vinda de outros antropólogos, e novas questões e interpretações teóricas, atualmente retomadas.
\end{abstract}

PALAVRAS-Chave: Escola de Chicago, Robert Park, Donald Pierson, relações raciais, Bahia e Brasil.

\section{INTRODUÇÃO}

Robert E. Park visitou o Brasil durante um mês e meio em 1937. Nessa época, ele se encontrava na Universidade de Fisk (Tennessee) após ter se aposentado pela Universidade de Chicago, onde ensinou no Departamento de Sociologia de 1913 a

* Doutora em Sociologia. Professora na Université de Lille 1 (Franca) e membro do Centre Lillois d'Études et de Recherches Sociologiques et Economiques - CLERSE/CNRS. Pesquisadora associada ao IUPERJ, Rio de Janeiro (Brasil).

Faculté des Sciences Economiques et Sociales. Université des Sciences et Technologies de Lille. 59655. Villeneuve d'Ascq Cedex. France. licia.valladares@univ-lille1.fr.

A autora agradece a Mariza Corrêa, Gilberto Velho e a Anete B. L. Ivo, primeiros incentivadores deste estudo, e a Cecília Sepúlveda, Cláudia Cruz e Lerice Grazoni que, na qualidade de assistentes de pesquisa, colheram dados em Salvador, na Biblioteca Nacional, no Arquivo Nacional do Rio de Janeiro e no Arquivo Edgard Leuenroth da UNICAMP. Agradecimentos vão também para a Diretora da Casa de Oliveira Vianna, em Niterói, Rio de Janeiro e a Maria Brandão (em Salvador) pelo arquivo de cartas de Thales de Azevedo. Nos Estados Unidos, várias pessoas foram extremamente simpáticas, dentre as quais: James Grossman, editor da Encyclopedia of Chicago e diretor da Newberry Library; Julia Gardner e Barbara Gilbert da Special Collections Research Center, University of Chicago Library; Dani Smith, diretora do Department of Social Science, Fisk University e Beth Howse do Archival and Manuscript Collections of the Fisk University, Franklin Library. William Kornblum, do Graduate Center, City University of New York e Diana Brown, Bard College, que foram interlocutores constantes. Anna Sant'Anna, do Lincoln Institute of Land Policy de Harvard, intermediou
1933. Ele veio ao Brasil acompanhado de sua esposa, Clara Cahill Park. O principal motivo dessa viagem era o de supervisionar o trabalho de campo de Donald Pierson, ${ }^{1}$ seu aluno de PhD da Universidade de Chicago, que estava morando em Salvador com sua esposa americana, desde 1935, estudando relações entre brancos e pretos na Bahia. ${ }^{2}$

a relação com Lisa Redfield Peattie, neta do Robert Park. Sou também grata a Peter Ward, professor do Department of Sociology, University of Texas at Austin e a Bryan Roberts, então diretor do Teresa Lozano Institute of Latin American Studies, que me convidaram como visiting ressource professor a Austin. Finalmente, não posso deixar de mencionar Edmond Préteceille, que acompanhou com enorme interesse minhas "aventuras" pelos arquivos americanos.

* * Tradução da documentação citada, do inglês para o português, de Any B. Leal Ivo, com revisão da autora.

${ }^{1}$ Eram membros do Committee responsável pela tese de Donald Pierson nada menos que Robert Redfield, Louis Wirth e Robert Park. Robert Redfield, na qualidade de presidente do Committe, é quem deveria ter vindo ao Brasil. Charles Johnson, da Universidade de Fisk, diretor do Departamento de Ciências Sociais, também cogitara em vir, mas foi forçado a desistir por conta de sua agenda de compromissos. Fonte: Carta do Park ao Pierson, em 7 de abril de1937, anunciando sua visita à Salvador (Arquivo Edgard Leuenroth).

${ }^{2}$ Mariza Corrêa, em História da Antropologia no Brasil (19301960), colheu o testemunho de Donald Pierson, oferecendo, em "Algumas atividades no Brasil em prol da antropologia e outras ciências sociais", os principais momentos que marcaram sua longa trajetória de dezoito anos no Brasil. Várias das informações aqui reproduzidas sobre Donald Pierson foram tiradas desse seu depoimento a Corrêa. 
Após ter passado julho e agosto de 1937 no Brasil, primeiro no Rio de Janeiro, quando se encontrou com Artur Ramos, ${ }^{3}$ e, em seguida, em Salvador, onde foi apresentado por Donald Pierson à cidade, à sua rede de relações e aos candomblés baianos, Robert Park retornou à Tennessee, onde, desde 1936, atuava como professor convidado da Universidade de Fisk, a convite de seu ex-aluno Charles Johnson, ${ }^{4}$ então Diretor do Departamento de Ciências Sociais. Nessa universidade (à época exclusiva para estudantes negros), Robert Park ministrou, junto com Donald Pierson, ${ }^{5}$ em 1938, um seminário sobre Raça e Cultura. Em 1942, a tese de Donald Pierson Negroes in Brazil: a study of race contact at Bahia foi publicada pela University of Chicago Press, com uma introdução de Robert Park. O trabalho recebeu o prêmio Anisfield, outorgado pela Social Science Research Council como "o melhor livro científico do ano sobre relações de raça no mundo contemporâneo". ${ }^{6} \mathrm{O}$ livro foi publicado em português sob o título de Brancos e Prêtos na Bahia. Estudo de contato racial, em 1945, pela CompanhiaEditora Nacional, acompanhado de duas introduções, uma de Robert Park e a outra de Arthur Ramos e republicado, nos Estados Unidos, em 1966, pela Southern Illinois University Press e, no Brasil, em 1971, com uma nova introdução, revista e atualizada pelo autor pela Editora Nacional, série 241, Brasiliana.

A viagem deRobertParkaoBrasilépouquíssimo

conhecida dos autores americanos que escreveram sobre ele e sua obra. Somente Matthews (1977),

${ }^{3}$ Fonte: Carta do Robert Park a Arthur Ramos, escrita de Salvador, em 19 de agosto de 1937 (Biblioteca Nacional, Arquivo Arthur Ramos I-35,36,2.032). Nessa carta, Park faz menção ao Hugh Tucker, americano que vivia há muitos anos no Rio e com quem foi até a residência de Arthur Ramos.

${ }^{4}$ Charles Johnson havia sido aluno do Park em Chicago. Em 1922, escreveu o livro The negro in Chicago. A study of race relations and a race riot in 1919. Ver Referências.

${ }^{5}$ Donald Pierson foi, a convite do Robert Park, para Tennessee, após sua estadia na Bahia. Robert Park conseguira para ele uma posição de assistente na Universidade de Fisk. (Fonte: Carta do Robert Park a Charles Johnson, então chefe do Departamento de Sociologia, Archival and Manuscript Collections of the Fisk University Franklin Library). Os Pierson's moraram em Nashville com o casal Park, enquanto Donald Pierson redigia a sua tese de doutorado. Trata-se do único caso em que um aluno de PhD de Robert Park morou com ele. Raushenbush (1979) menciona o fato na biografia que escreveu sobre Park.

${ }^{6}$ Informação dada pelo próprio Pierson a Corrêa (1987, p.40).
Raushenbush (1979) e Coser (1971) a mencionam, mas sem lhe atribuir qualquer importância. ${ }^{7}$ É como se, para a trajetória intelectual de Park, a viagem ao Brasil importasse pouco. ${ }^{8}$ Com efeito, Raushenbush (1979, p. 197-198), responsável pela principal biografia do autor, não menciona, entre as introduções feitas por Park aos livros de seus orientandos, aquela escrita para o livro do Donald Pierson! Republicada essa introdução em Race and Culture (1950), sob o título de The career of the Africans in Brazil, o capítulo não interessou aos schollars [estudiosos] americanos. ${ }^{9} \mathrm{~A}$ grande maioria dos livros e artigos escritos sobre Park versa sobre a sua contribuição para a sociologia americana, destacando dois períodos: aquele que antecedeu a sua ida para Chicago, quando Park era jornalista, e o período em que Park, na Universidade de Chicago, esteve à frente da corrente que viria a se chamar de "Escola de Chicago" ${ }^{10}$

Neste artigo, procuro mostrar a importância da viagem de Park ao Brasil no final dos anos 30 , sugerindo que foi a partir de então que a ciência social internacional descobriu a Bahia, trans-

O mesmo pode ser dito em relação aos autores franceses. Chapoulie (2001), que escreveu o mais completo livro em língua francesa sobre a tradição sociológica de Chicago, menciona Donald Pierson, mas não faz referência à viagem de Park ao Brasil. No seu livro sobre A Escola de Chicago (1992, traduzido para o português em 1995), publicado na coleção Que Sais-je?, Coulon desconhece Donald Pierson, nẩo fazendo qualquer menção à viagem de Park ao Brasil.

${ }^{8}$ A neta de Park, Lisa Redfield Peattie, com quem conversei longamente, não sabia que seu avô estivera no Brasil e na Bahia! O mesmo pode ser dito de vários sociólogos urbanos que encontrei nos Estados Unidos (nas Universidades de Harvard, Brown, City University of New York, Columbia, Princeton, Austin), durante a minha pesquisa entre outubro de 2008 e fevereiro de 2009.

${ }^{9}$ Robert Park só escreveu sobre o Brasil uma vez. Não encontrei o artigo prometido à revista África, intitulado "Relics of The Persistance of African Culture in Bahia". Diz D. Westermann, do Internationales Institut fur afrikanische Sprachen und Kulturen, em carta ao Park, datada de 29 de Outubro de 1937: I should like to see the ms of your article on Relics of the Persistence of African Culture in Bahia. If it is not too long and not too far away from the aims which our journal pursues, it might be possible to publish it. So if you will kindly send dit, I shall have pleasure in examining it [Eu gostaria de ver o ms [manuscrito] do seu artigo sobre Relíquias da Persistência da Cultura Africana na Bahia. Se não for muito longo nem muito distante dos propósitos que a nossa revista adota, é possível publicá-lo. Então, se você cordialmente me enviar, eu terei prazer em examinálo].(Fonte:Archival and Manuscript Collections of the Fisk University Franklin Library). Tal artigo, aparentemente, não chegou a ser publicado.

${ }^{10}$ Nas minhas buscas em bases bibliográficas nos Estados Unidos, encontrei apenas um artigo sobre Robert Park em Fisk. Ver Cahnmann (1978). 
formada desde então em um "laboratório social". Trata-se de uma pequena contribuição à história das ciências sociais no Brasil, pois me interesso pela circulação das ideias e pela recepção da "Escola de Chicago" entre nós. ${ }^{11}$

\section{QUEM ERA O ROBERT PARK QUE VISITOU O BRASIL? O QUE O TROUXE ATÉ AQUI?}

Robert Park veio ao Brasil em 1937, quando já estava com 73 anos (nascera em 1864, na Pennsylvania) e já conhecia boa parte do mundo, sendo um autêntico world wide traveler [homem do mundo]. Diferentemente de seus conterrâneos americanos da época, estivera na Europa e em outros continentes. Como estudante, em Berlim (onde frequentou cursos dados por Simmel), em Strasburgo ${ }^{12}$ e em Heildelberg, onde defendeu sua tese de doutorado em 1903, The Crowd and the Public. ${ }^{13}$ Como jornalista, acompanhando olíder negro Booker T. Washington em sua viagem pela Europa, ajudouo a escrever The Man Farthest Down (1912). ${ }^{14} \mathrm{Na}$ qualidade de professor, esteve por bastante tempo em Honolulu e Hawai (onde coordenou o Survey of Race relations in the Pacific Coast), na China (onde foi professor convidado durante três meses em Peiping), no Japão, nas Philipinas, na Indonésia, na África do Sul. Nessas viagens de volta ao mundo, se fez sempre acompanhar de Mrs. Park e foi em sua companhia que desembarcou no Rio de Janeiro, do navio Southern Cross, em 15 julho de 1937, ${ }^{15}$ antes

${ }^{11}$ Um primeiro interesse no estudo da recepção da Escola de Chicago no Brasil está registrado em Valladares (2005), onde já é feita uma menção à visita do Robert Park à Bahia. Há vários trabalhos sobre a importância de Donald Pierson na sociologia brasileira através dos dezesseis anos em que foi professor na Escola Livre de Sociologia e Política de São Paulo: Durham e Cardoso (1961); Corrêa (1987); Limongi (1989); Massi (1989); Vila Nova (1998); Mendoza (2005), entre inúmeros outros.

${ }^{12}$ Sobre seus dois anos e meio passados em Strasbourgo, ver Denis in Guth (2008).

${ }^{13} \mathrm{O}$ título original de sua tese em alemão era Masse und Publikum. Sobre essa tese, escrita sob a orientação de Wilhlem Windelband, ver o artigo de Guth in Guth (2008).

${ }^{14}$ Segundo Coser (1971) e vários outros autores americanos. Na ultima edição desse livro, consta Robert Park como co-autor.

${ }^{15}$ Fonte: Informação colhida junto ao Arquivo Nacional. Fundo DPMAF, livro RV397. de seguir para Salvador.

Park vem, portanto, ao Brasil num momento de sua vida em que já é um sociólogo reconhecido tanto dentro como fora dos Estados Unidos. Publicara, juntamente com Ernest Burgess, em 1921, Introduction to the Science of Sociology, coletânea apelidada de Green Bible [Bíblia Verde], tal foi sua importância à época. ${ }^{16}$ Fora eleito, em 1925 , presidente da American Sociological Society e era membro do conselho editorial de várias revistas científicas. Suas ideias sobre a ecologia urbana eram amplamente difundidas, e seu artigo Suggestions for the investigation of human nature in the urban environment, publicado no livro The City (1925), editado junto com Ernest Burgess, tornou-se um clássico para se pensar a cidade. Inúmeros dos seus alunos de $\mathrm{PhD}$ em Chicago tiveram suas teses publicadas, dentre as quais se tornaram clássicos os estudos de Nels Anderson (1923), Thrasher (1927), Louis Wirth (1929), Zorbaugh (1929), Shaw (1930) e Stonequist (1937), dentre outros.

Robert Park já era conhecido dos principais sociólogos e antropólogos brasileiros quando aqui desembarcou em 1937, o mesmo podendo ser dito da Escola de Chicago, da qual Robert Park tornarase figura chave. Gilberto Freyre, que estudara nos Estados Unidos (na Columbia, New York, nos anos 1920) começa o seu livro Nordeste, aspectos da influencia da canna sobre a vida e a paisagem do nordeste do Brasil (1937), anunciando: "Este ensaio é uma tentativa de estudo ecológico do Nordeste do Brasil" (grifo nosso) e, em nota de pé de página, cita os principais autores da nova bibliografia que "já inclue trabalhos de valor" (Freyre, 1937, p. 9-10), dentre os quais os de McKenzie e os de Robert Park. Outro brasileiro de renome, Oliveira Vianna, já havia entrado em contacto com Park e lhe enviado seus livros Raça e Assimilação, publicado em 1932, e Populações Meridionais do Brasil, cujo primeiro volume é de $1920 .{ }^{17}$ Arthur Ramos, na introdução que escreveu ao livro do ${ }^{16}$ Ver Schrecker in Guth (2008).

${ }^{17}$ Carta de Robert Park a Oliveira Vianna, datada de 13 de julho de 1933. Nessa carta, Park agradece o recebimento dos livros dedicados a ele por Oliveira Vianna. (Fonte: Biblioteca Oliveira Viana, Casa de Oliveira Vianna, Niterói, Reg. $\mathrm{n}^{\circ}$ 1035.1). 
Donald Pierson, diz que o “jovem sociólogo vinha formado dentro da rígida disciplina metodológica da sua universidade onde recebeu os ensinamentos do grande Park" (Ramos, 1943. grifo nosso). Um autor contemporâneo (Vila Nova, 1998) lembra-nos que a criação da Escola Livre de Sociologia e Política de São Paulo esteve vinculada a uma preocupação das elites com uma eficiente atuação na vida social e com a "confiança iluminista na ciência como instrumento seguro de reforma social" (p. 119). Segundo Vila Nova (1998), os autores do projeto da Escola vão na direção do pragmatismonaturalista, no sentido desenvolvido pelos sociólogos de Chicago. ${ }^{18}$ Vila Nova chega a afirmar que "Park exerceu influência significativa sobre Freyre quanto à sua concepção da sociologia como ciência mista ou anfíbia. É que a sociologia vem sendo ao mesmo tempo ciência natural e, com outras ciências chamadas sociais, ciência cultural" (Vila Nova, 1998, p. 125).

Muito embora uma "obrigação universitária" teria sido a causa primeira da viagem de Park ao Brasil, em 1937, o que parece tê-lo trazido até aqui foi, com efeito, o seu interesse pelas relações raciais. Nos seus tempos de jornalista, em que fora secretário da Congo Reform Association e assessor por sete anos de Booker T. Washington no Tuskegee Institute no Alabama (antes, portanto, de se tornar professor universitário), Robert Park conheceu, entrevistou e conversou com centenas

${ }^{18}$ Não por coincidência Donald Pierson é convidado em 1939 a integrar o corpo docente da Escola Livre de Sociologia e Política de São Paulo.

${ }^{19}$ Park diferenciava os processos de competição, conflito, acomodação e assimilação. Ver Coser (1971). rente entre os dois grupos. Os negros tiveram de reinterpretar a cultura anglo-saxã (apesar de serem isolados do mundo dos brancos), enquanto que os europeus trouxeram consigo a sua cultura e os seus valores.

O contato entre raças e entre culturas era, de fato, o que interessava a Robert Park. Não por acaso seu livro póstumo, publicado em $1950 \mathrm{com}$ um prefácio de Everett Hugues, se chama Race and Culture. Não por acaso também, quando Park se aposenta pela universidade de Chicago, vai para a Universidade de Fisk, uma universidade negra. Sua opção por Fisk tem a ver com a relação próxima com seu ex-aluno Charles Johnson (à época, chefe do Departamento de Sociologia), mas também representa uma escolha consciente e clara. São palavras do próprio Park:

\begin{abstract}
My primary purpose in coming to Fisk was (1) to renew my acquaitance with the South and (2) in view of the fact that I had long been interested in racial studies, explore the possibility of an institute for the systematic study of race problems. [Meu propósito original vindo para Fisk foi (1) renovar minha familiaridade com o Sul e, (2) tendo em vista que eu tenho estado há muito tempo interessado por estudos raciais, explorar a possibilidade de um instituto para o estudo sistemático dos problemas raciais]. ${ }^{20}$
\end{abstract}

Apesar de ter seu nome associado à sociologia urbana, trazendo para a universidade seu interesse pela cidade, vista não tanto pela ótica geográfica, mas como um “organismo social”, Park nunca deixou de se interessar pelas questões do contato racial e cultural, considerando-os cada vez mais numa perspectiva internacional. Suas estadias na China, na Índia, no Japão, em Havaí e na África do Sul serviriam não apenas para ampliar sua rede de relações universitárias, como também para verificar suas ideias e conceitos nos diferentes contextos. E, no dizer de Charles Johnson (1945):

Just as he found in race and color in America an index to human relations, so race and culture problems throughout the world gave him a key to the understanding of the process of civilization.

${ }^{0}$ Documento escrito por Robert Park "Comments of Graduate Study at Fisk University", datado de 19 de outubro de 1938. (Fonte: Archival and Manuscript Collections of the Fisk University Franklin Library). 
[Assim como ele encontrou na raça e cor na América um registro das relações humanas, do mesmo modo os problemas de raça e cultura através do mundo deram-lhe a chave para entender o processo de civilização].

O Brasil seria, portanto, mais um ponto de referência no contexto internacional, embora dotado de uma dimensão própria. Lembremos que Pierson fala de uma viagem de volta ao mundo em 1934, durante a qual Park teria visitado a América do Sul. Não encontramos, nos arquivos americanos consultados, traços dessa sua passagem pelo Brasil em 1934, mas Park aqui esteve, ${ }^{21}$ embora muito rapidamente, e foi nessa primeira viagem que conheceu, no Rio de Janeiro, Artur Ramos e Oliveira Vianna (Corrêa, 1987, p. 36-37) e que teria adquirido o livro de Nina Rodrigues Os Africanos no Brasil (Corrêa, 1987, p. 35).

Com efeito, o Brasil era conhecido dos acadêmicos estrangeiros por ser uma região que diferia do modelo americano de relações raciais e culturais. Gilberto Freire havia publicado em 1922, no volume 5 da Hispanic American Historical Review, a sua dissertação de mestrado "Social Life in Brazil in the middle of the XIX century", germe embrionário de Casa-grande e Senzala, publicado pela primeira vez em 1933 (TUNA, 2008). Nina Rodrigues era, já à época, referência obrigatória dos estudos sobre o negro no Brasil. Dois Congressos Afro-Brasileiros haviam sido organizados, no Recife e em Salvador, respectivamente em 1934 e 1937, contando ambos com a participação de brasileiros (acadêmicos e pessoas ligadas aos candomblés) e um ou outro estrangeiro. ${ }^{22} \mathrm{O}$ Brasil era, sem dúvida, um caso interessante sobre essa temática, que

${ }^{21}$ Conforme o Pierson atesta em entrevista concedida a Corrêa (1987).

${ }^{22}$ O primeiro congresso, de 1934, foi organizado por Gilberto Freyre no Recife, e o segundo foi organizado por Edison Carneiro, em Salvador, em 1937, meses antes da visita do Park. Arthur Ramos participou do primeiro congresso, Donald Pierson participou do segundo, muito embora se previsse também a participação de outro estrangeiro (foi o caso de Herskovitz) que não compareceu. Sobre o primeiro Congresso Afro-brasileiro, ver Levine (1973: 191), que diz: The fact that the Congress identified Afro-Brazilian studies as a subject worthy of study, recognizing the multi-faceted nature of the subject and approaching it from a wide range of disciplines is significant in its own right. $\mathrm{O}$ fato de que o Congresso ter identificado os estudos Afro-Brasileiros como um assunto digno de estudo, reconhecendo a natureza multifacetada da temática e abordando-a através de uma merecia ser estudado e mais conhecido, diferenciando-se substancialmente do caso norte-americano por basear-se na miscigenação.

Foi certamente sabendo que o Brasil apresentava uma configuração particular de difícil reconciliação com as ideias sobre as relações raciais nos Estados Unidos que Robert Park sugeriu a Donald Pierson que viesse aqui estudar a "situação racial”. ${ }^{23}$ E foi, sem dúvida, querendo ver com os próprios olhos uma situação onde o melting pot $^{24}$ de raças e culturas acabava por desembocar na miscigenação, que planejou com sua esposa uma longa estadia na Bahia em 1937.

\section{O "Homem Marginal"}

Quando chegou a Salvador, Park já era o "conhecido e famoso americano Robert Park" da Universidade de Chicago.

Em 1928, Park publicara um artigo na American Journal of Sociology, intitulado "Human Migration and the Marginal Man”. Este artigo viria a contribuir para uma discussão muito presente à época sobre a integração dos imigrantes na sociedade norte-americana e consistiria numa das principais contribuições de Robert Park para a história da sociologia americana (Coser, 1971).

A noção de homem marginal, quando primeiramente cunhada, não é negativa, como poderia parecer. No artigo em questão, Park desenvolve as ideias de Simmel, ${ }^{25}$ baseando-se na figura do "estrangeiro" [The Stranger]. O estrangeiro é aquele que,

ampla série de disciplinas é em si significativo.].Ver também Romo (2007) que, mais recentemente, escreveu sobre o mesmo congresso.

${ }^{23}$ Pierson, no prefácio à primeira edição norte-americana do seu livro Brancos e Pretos na Bahia, diz: "Robert E. Park voltava de uma longa viagem pelo mundo, durante a qual tinha observado in loco alguns dos mais importantes centros de contato racial e cultural, inclusive o Brasil” (Pierson, 1971, p. 76, 2 a edição).

${ }^{24}$ N. da Editora: Segundo a autora (ver adiante) Chicago havia sido caracterizada por Park e seus discípulos como um "melting pot", onde conviviam diferentes comunidades pertencentes a diversas nacionalidades, mas que não se misturavam.

${ }^{25}$ Park havia estudado com o Simmel na sua passagem por Berlim e é considerado o introdutor de Simmel nos Estados Unidos, tendo incluído vários de seus artigos na coletânea Introduction to the Science of Sociology (1921). 
vindo de fora, fica amanhã. Ele se instala na comunidade, mas fica à sua margem, permanecendo, de alguma maneira, exterior ao grupo social. Desenvolve uma personalidade marginal na medida em queé um homem à margem de duas culturas e duas sociedades. Segundo Simmel, o judeu emancipado é tipicamente um homem marginal. Eleé, por excelência, um "estrangeiro", um cosmopolita. Park, baseando-se em Simmel, mas também em autobiografias de imigrantes judeus publicadas nos Estados Unidos, nos diz que todas são versões de uma mesma história: a história do homem marginal, daquele que migrou para os Estados Unidos e está buscando um lugar numa cidade que é mais livre, mais complexae mais cosmopolita do que o seu local de origem. Nessas autobiografias, o conflito de culturas é o conflito do self dividido [divided self], do velho e do novo self. Para Park, portanto, o homem marginal é o produto de conflitos interculturais.

A partir da tese de seu aluno Stonequist (1937), Park dará mais tarde outro sentido à expressão homem marginal, que passará a ter uma conotação mais negativa, incluindo a situação dos negros do Sul dos Estados Unidos, que vivem à margem da cultura branca. O homem marginal será tipicamente um imigrante da segunda geração, que sofre os efeitos da desorganização do grupo familiar, como a delinquência juvenil, a criminalidade, o divórcio. Livre de seus antigos valores e tradições, ele tem sua antiga identidade afetada, mas encontra-se ainda sem orientação diante dos novos valores da sociedade que o acolhe... Nessa sua nova concepção, o homem marginal continua sendo um migrante, porém ele pode ser representado por um europeu ou um negro do Sul dos Estados Unidos que veio à cidade em busca de trabalho, ou ainda um camponês americano que sofreu os efeitos do êxodo rural. O homem marginal é alguém que, ao separar-se de sua cultura de origem, constrói, no processo de aculturação, uma nova identidade.

Para Park, portanto, o homem marginal é um híbrido cultural, que se encontra entre duas culturas distintas, não sendo plenamente aceito por nenhuma delas. No entanto, a mestiçagem não deixa de ser um enriquecimento, e o homem marginal é suscetí- vel de criatividade, ligada ao fato de sua posição crítica em relação ao meio que não lhe é familiar.

Park certamente chegou ao Brasil e à Bahia querendo testar essas ideias... Como todo intelectual, vinha imbuído de pensamentos e esquemas provenientes das realidades com as quais se vira confrontado. ${ }^{26}$

\section{Um Melting Pot baiano?}

Embora tendo vindo ao Brasil de Nashville, onde vivia e ensinava, não há dúvidas de que, para Park, Chicago era a referência do que seria uma metrópole moderna, uma "cidade".

Chicago havia sido caracterizada por Park e seus discípulos como um melting pot, onde conviviam diferentes comunidades pertencentes a diversas nacionalidades, mas que não se misturavam. Halbwachs, sociólogo francês, em visita a Chicago nos anos $1930,{ }^{27}$ espantou-se com a quantidade de estrangeiros aí presentes: poloneses, alemães, russos, italianos, gregos, tchecos, irlandeses, suecos, eslavos, judeus de diversas procedências, totalizando mais de vinte nacionalidades que viviam numa mesma cidade, distribuídos em várias comunidades (Halbwachs, 1932). Chicago, com certeza, era uma cidade de estrangeiros, e eles correspondiam, na mesma época, a cerca de $70 \%$ da população local. Jane Addams, pioneira da filantropia em Chicago desde o final do século XIX, no livro Hull House Maps and Papers mostra, rua por rua, a concentração dos diversos imigrantes. ${ }^{28}$ Porém, à diferença segundo a nacionalidade so-

${ }^{26}$ Na leitura de Coulon (1995): “... se alguns grupos sociais permanecem marginalizados e desenvolvem culturas intermediárias, híbridas, sem jamais se assimilar totalmente à cultura dominante, é porque o homem marginal possui uma ambiguidade fundamental. Mesmo sendo um homem criativo, que inventa novas formas de sociabilidade e novos traços culturais, é também o que sofre esta situação dual de maneira dolorosa, com manifestações psicológicas que ao mesmo tempo o revelam e o designam como um desviante social".

${ }_{27}$ Ver Topalov (2007), que descreve e analisa Halbwachs em Chicago, em 1930.

${ }^{28}$ Quanto à população negra de Chicago, ela somente teve peso a partir de 1914, quando começaram a chegar os negros vindos dos estados do Sul. Em 1930, a população negra correspondia a $7 \%$ da população total (Halbwachs, 1932) 
brepunha-se uma diferença de classe social, o que dava à cidade uma configuração espacial bastante especial. Tinha-se assim, em Chicago, uma aglutinação dos migrantes recentes e ainda não "integrados" à sociedade americana nas Little Italy ou China Town ou no Ghetto Judaico (conhecido como The Ghetto), localizados nas áreas centrais da metrópole. Verdadeiras "zonas morais", tais territórios (verdadeiros enclaves) supriam as funções de proteção diante do novo ambiente e ajudavam os diversos grupos étnicos na manutenção da tradição e na reprodução de valores.

Uma vez "integrados" à sociedade, os "novos americanos" iam para a periferia, onde habitavam em bungalows [bangalôs] ou em bairros exclusivos. A mobilidade residencial correspondia, então, à mobilidade social. O esquema de Burgess e Park (inspirado por Chicago) da cidade em zonas concêntricas (Park, Burgess e Mackenzie, 1925) refletia bem essa dinâmica social e espacial: à medida em que se progredia na escala social, as áreas centrais da cidade eram deixadas em prol da periferia.

A Salvador que Park encontrou em 1937 não correspondia ao modelo ideal de cidade inspirado pelo caso de Chicago. Havia apenas 290.443 habitantes no município de Salvador em 1940, não sendo, portanto, Salvador, àquela época, uma metrópole. Por outro lado, o Censo de 1940 nos informa que Salvador não tinha uma população estrangeira substancial. Em 1940, os estrangeiros totalizavam apenas 5.439 habitantes (quase $2 \%$ da população total). E, dentre os estrangeiros, a colônia espanhola era a mais numerosa, ${ }^{29}$ não havendo, no entanto, uma concentração deles em uma só zona da cidade. Os espanhóis se encontravam dispersos nos diferentes bairros de classe média. Por outro lado, a migração para Salvador era, sobretudo, de baianos que se deslocavam do interior para a capital. A população não era, portanto, composta de estrangeiros, mas de baianos e de todos os matizes, fruto da sua composição inter-racial. Homem marginal? Melting pot?

A composição da população de Salvador,

${ }^{29}$ Informação proveniente da Enciclopédia dos Municípios Brasileiros, XXI Volume, Rio de Janeiro. segundo a cor, no Recenseamento de 1940, era a seguinte:

\begin{tabular}{l|r|c}
\hline Cor & Número & \% \\
\hline Branca & 101.892 & 35,08 \\
\hline Preta & 76.472 & 26,33 \\
\hline Parda & 111.674 & 38,45 \\
\hline Amarela & 146 & 0,05 \\
\hline Cor não declarada & 259 & 0,09 \\
\hline Total & 290.443 & 100,00 \\
\hline
\end{tabular}

Fonte: IBGE. Censo Demográfico: população e habitação de 1940. [Elaboração própria]

Onde estava, portanto, o melting pot? O caso baiano era de miscigenação, como mostravam os dados de 1940 (38\% da população se declarou parda $^{30}$ ) e como reconheceu Donald Pierson (1971):

... a miscigenação se tem processado na Bahia ininterruptamente e sem provocar atenção sobre si durante longo período de tempo. Talvez em poucos lugares do mundo o cruzamento interracial se tenha dado de maneira tão contínua e em escala tão extensiva em tempos recentes. (p.362).

A cidade do Salvador se encontrava dividida em diferentes espaços étnicos, mas a divisão da cidade estava também marcada por espaços ricos e espaços pobres. Nas palavras do próprio Park (Pierson, 1971, p. p.84):

... para o estrangeiro que na Bahia percorra uma
das elevações onde moram os ricos, é uma expe-
riência um tanto bizarra, ouvir, vindo dentre as
palmeiras dos vales vizinhos, onde os pobres
moram, o insistente rufar dos tambores africa-
nos. Tão estreitas são as distâncias espaciais que
separam a Europa, situada nas elevacóes, da Âfri-
ca, situada nos vales, que é dif́cil perceber a
amplitude das distâncias sociais que as
separam. (grifos nossos)

O que chamou a atenção de Park foi, portanto, a divisão de classes existente na sociedade baiana, que se expressava também por uma divisão étnica e de ocupação do espaço urbano.

Park não ficou, porém, imune à miscigenação que constatou na sua visita a Salvador e que lhe foi mostrada por Donald Pierson ao percorre-

${ }^{30}$ Em 1950, segundo o Recenseamento, a população que se definia como parda em Salvador já atingia $41 \%$ da população total. 
rem as ruas da cidade, ao visitarem pessoas da sociedade baiana, ao assistirem a várias cerimônias em terreiros de candomblé. A miscigenação, uma característica do Brasil, não era nada frequente nos Estados Unidos de então, onde a ideologia racial tendia a se perpetuar, impossibilitando a mistura racial. ${ }^{31}$ Park reconhecia as dificuldades de um pesquisador estrangeiro (americano) diante de tal situação. São palavras do próprio Park:

Mais difícil ainda para nós, cuja concepção do problema do negro e das 'relações raciais' se formou nos Estados Unidos, é compreender em todas as suas minúcias a 'situação racial' num país de história e tradição diferentes. (Pierson, 1971, p. 84-85).

O que a miscigenação representava no Brasil e na Bahia?

Park chegou mesmo a repensar o significado do melting-pot e escreveu, na Introdução do livro de Pierson (1971, p. 82):

Ao sugerir a possibilidade de estudos futuros em seguida a este, estou levando em conta o seguinte: 1) que o Brasil é um dos mais importantes "melting-pots" de raças e culturas em todo o mundo, onde a miscigenação e aculturação estão se processando; e 2) que o estudo comparativo dos problemas de raça e cultura provavelmente assumirá uma importância especial nesta época, em que a estrutura de ordem mundial parece estar se desintegrando devido à dissolução das distâncias físicas e sociais, sobre as quais esta ordem parece repousar.

A visita de Park a Bahia teve, ao que parece, consequências na sua maneira de pensar a questão racial... ${ }^{32}$

\section{Bahia: um laboratório social}

O Brasil era considerado pelos scholars americanos um verdadeiro "laboratório da civilização", afirmaria Arthur Ramos em 1943, na introdução à primeira edição do livro do livro do Donald Pierson. A referência era ao Brasil como um todo, mas não a Salvador, na Bahia, em particular. Defenderemos

${ }^{31}$ São palavras de Park na Introdução do livro do Pierson 1971, p. 83): Fato que torna interessante a "situação racial" brasileira, é que tendo uma população de côr proporcionalmente maior que a dos Estados Unidos, o Brasil não tem "problema racial"

${ }^{32}$ A desenvolver em outro artigo. aqui a hipótese de que foi somente após a estadia do Donald Pierson e da visita de Robert Park a Salvador que essa cidade seria "transformada" em um verdadeiro laboratório social. ${ }^{33}$

A cidade como laboratório foi uma das principais contribuições de Robert Park à sociologia urbana ou à ciência da cidade. ${ }^{34} \mathrm{~A}$ ideia primeira teria vindo de Albion Small, então chefe do Departamento de Sociologia. A imagem da cidade como laboratório foi, segundo Leclerc (1979), uma forma publicitária que Park encontrara para explorar o material rico que as cidades americanas ofereciam para que se analisassem os problemas de pobreza, da integração e das formas de organização social da sociedade. Não interessava simplesmente o estudo da cidade, mas a compreensão científica de seus problemas que, em consequência do rápido crescimento demográfico, da forte presença de imigrantes europeus, da intensificação do conflito entre capital e trabalho, eram inúmeros.

Como a época era de estabelecimento dos princípios científicos da sociologia, a metáfora do laboratório fazia sentido. Como explicar, por exemplo, a anatomia da vida coletiva? Uma morfologia que se transformava rapidamente e que, a olho nu, sugeria uma evolução da vida urbana, da vida dos grupos, das comunidades? A cidade não seria como um laboratório na medida em que ela constituiria um dispositivo de controle das condições sociais de reprodução do comportamento humano?

Salvador foi, então, "transformada" (como o fizera com Chicago, Park e seus discípulos) em um laboratório social. Iniciando-se pela estadia de Pierson entre 1935 e 1937. Durante 16 meses, o casal Pierson residiu na Vitória, na Barra e nas Mercês (bairros à época de classe alta e média) e no Rio Vermelho (que, segundo Pierson, na época, era um bairro pobre, onde se encontravam os principais candomblés da Bahia). ${ }^{35}$ Pierson fez tra-

${ }^{33}$ Inicialmente, para os pesquisadores americanos.

${ }^{34}$ Ver, nas Referências, o artigo do Park intitulado "The city as a social laboratory", publicado em 1929 na coletânea Chicago: an experiment in social science research.

${ }^{35}$ Fonte: Carta de Donald Pierson a Robert Park, datada de 24 de Fevereiro de 1937. Pierson fala do Rio Vermelho como área que dava acesso ao Engenho Velho, Matta Escura, Garcia e Gantois. Archival and Manuscript Collections of the Fisk University Franklin Library. 
balho de campo em toda a cidade. Seguindo os ensinamentos do Park, "vasculhou" tudo o que existia à época: bibliografia cientifica em português, francês, inglês, alemão, notícias em jornais, material existente nas repartições públicas e no Instituto Geográfico e Histórico da Bahia e em outros arquivos (documentos históricos). Frequentou as festas populares de Salvador, seus clubes sociais, seus inúmeros candomblés. Anotou tudo que lhe parecia importante. Empregou a técnica do questionário, mas fez também entrevistas formais e informais, pediu a algumas pessoas que elaborassem listas. Enfim, aproveitou-se da rede de relações que estabeleceu, utilizando a técnica hoje conhecida como a de "bola de neve". ${ }^{36}$

Donald Pierson seguiu, portanto, o que o mestre propunha a seus alunos. ${ }^{37}$

Park, por sua vez, na sua estadia de quase dois meses em Salvador, também considerou a Bahia como um verdadeiro laboratório, onde tudo estava ainda por ser estudado cientificamente e onde o sociólogo ou antropólogo poderia se certificar ou não de suas ideias, ver em movimento os vários elementos de um conjunto, estudar a organização social da cidade e da sociedade. Com efeito, as situações encontradas por Pierson em Salvador eram bem diversas daquelas que o próprio Park havia encontrado em suas múltiplas andanças. A organização da sociedade, a ascensão social dos mestiços, a ruptura da antiga ordem baseada na escravidão, tudo isso o impressionou e o fez se perguntar se o mestiço baiano, brasileiro, correspondia ou não ao homem marginal que definira a partir dos Estados Unidos.

${ }^{36}$ Ver, no próprio livro do Pierson (Segunda edição, 1971), o Apêndice D, Estudo de Contacto Racial na Bahia: procedimento de pesquisa.

${ }^{37}$ É fato conhecido que Park dizia aos seus discípulos para se comportarem como um repórter. São palavras de Nels Anderson: De toutes les consignes données par Park celle-ci m'est restée en mémoire: 'Contentez-vous de retranscrire ce que vous voyez, ce que vous entendez et que vous savez, tout comme un journaliste'. [De todos os conselhos dados por Park este me ficou na memória: 'Contentai-vos em retranscrever o que virem, o que ouvem e o que sabem, como um jornalista'](Anderson, 1923, tradução francesa em 1993, p. 29). Pierson diz, em carta à Fred Matthews, datada de 7 Novembro de 1964: Dr. Park's advice to me before I left for Bahia was to put down everything that seems interesting or important. [Dr. Park me aconselhou a registrar tudo que parecesse interessante ou importante antes mesmo que eu partisse para a Bahia.]. (Fonte: Special Collections, University of Chicago Library, Robert Park Papers, Box 19).
Pierson disse de Park, quando este esteve em Salvador:

... he poked his nose into everything, observing, pausing to ask questions and to talk with anyone whose work or other activity interested him at the moment.

[... ele metia o nariz em todo lugar, observando, parando para perguntar e falar com qualquer pessoa cujo trabalho ou outra atividade o interessasse no momento]. ${ }^{38}$

Referindo-se, ainda, a Park, diz:

Perhaps what impressed me most about him was his constant and absorbing interest in and curiosity about all kinds and conditions of people and in the ways in which they live.

[Talvez o que mais me impressionou nele foi seu constante e absorvente interesse e curiosidade sobre todo tipo de pessoas e o modo como elas vivem. $]^{39}$

A esposa de Pierson também se refere à atitude do Park sobre Salvador:

Dr. Park and Donald roamed the city, talking with all kinds of people as they wandered through the fascinating streets and by-ways and out into the outlying portions of the city where the huts of the poor, including many Negro families, were located.

[Dr. Park e Donald percorriam a cidade, falando com toda espécie de pessoa, enquanto perambulavam por ruas fascinantes e ruelas situadas nas partes remotas onde os casebres dos mais pobres, incluindo os de muitas famílias negras, estavam localizados. $]^{40}$

De volta aos Estados Unidos, em fins de agosto de 1937, Park regressou à Universidade de Fisk, onde ofereceu, juntamente com Donald Pierson, um seminário sobre Raça e Cultura ${ }^{41} \mathrm{em}$ que (supomos) apresentou boa parte da bibliografia da tese do Donald Pierson, ${ }^{42}$ baseada em grande parte em autores brasileiros.

${ }^{38}$ Carta do Donald Pierson à Fred Matthews. (Fonte: Special Collections, University of Chicago Library, Robert Park Papers, Box 19).

${ }^{39}$ Idem, ibidem

${ }^{40}$ Pierson, Helen - Recollections of life with Dr and Mrs Park (dact.) (Fonte: Special Collections, University of Chicago Library, Robert Park Papers, Box 19).

${ }^{41}$ Infelizmente, não encontramos nos arquivos da Universidade de Fisk nenhum traço desse Seminário.

${ }^{42}$ Ver bibliografia selecionada da primeira edição (sobre a Bahia e sobre o Brasil) (Pierson, 1971) 


\section{A continuação da história...}

Logo após a viagem de Park ao Brasil, dirigiram-se à Bahia uma série de pesquisadores americanos, seguidos de franceses. ${ }^{43}$ Fora descoberta a Bahia pela ciência social internacional! ${ }^{44}$ Mas, como veremos adiante, sociólogos e etnólogos não estavam interessados nas mesmas questões: enquanto os primeiros se perguntavam sobre as mudanças e as formas de interação que apontavam para o futuro (a mobilidade social, a "carreira” dos negros), os etnólogos interessavam-se pela permanência das formas sociais e pelas culturas tradicionais (a herança africana através do candomblé).

Logo em seguida a Donald Pierson e a Park, veio a antropóloga Ruth Landes, em 19381939. Sua “saga” baiana está descrita em The city of women, publicado pela primeira vez nos Es-

${ }^{43}$ Dentre os franceses, o mais conhecido é o Roger Bastide, que escreveu em 1958 "Le Candomblé de Bahia”. Sobre a obra de Bastide, que morou em São Paulo 16 anos, ver Peixoto (2000).

${ }^{44}$ Como já dito e redito por vários autores (ver Referências), antes dos americanos já havia brasileiros estudando a questão racial no Brasil (Nina Rodrigues, Arthur Ramos, Gilberto Freyre, Edison Carneiro, entre outros). Vários outros brasileiros, como Thales de Azevedo, Florestan Fernandes, Oracy Nogueira, etc., dedicaram-se também às relações raciais, cultura e identidade negra no Brasil nos anos 1940, 1950 e 1960.

${ }^{45}$ Sobre a passagem de Ruth Landes pela Fisk University, onde foi instrutora, ver Cunha (2004) que pesquisou longamente nos seus arquivos localizados no Smithsonian Institute.

${ }^{46}$ Em carta a Hugh Tucker, Robert Park escreve: She already had fund for her enterprise before she knew that Mr. Pierson had preceded her at Bahia studying in the same general field. I met her just as I was leaving for New York a year ago. I suggested she should get in touch with $\mathrm{Mr}$. Pierson when she returned and that he would be at Fisk University in the fall, thereupon she postponed her visit to Bahia until she had a chance to get acquainted with Mr. Pierson's investigations. She has been at Fisk for the last six months and is planning to sail on the 23rd of April for Rio. [Ela já tinha encontrado recursos para vir à Bahia antes mesmo de saber que Mr. Pierson a tinha precedido na Bahia, estudando temática semelhante. Eu a conheci exatamente quando ela estava partindo para New York um ano atrás. Eu lhe sugeri entrar em contato com Mr. Pierson quando ela retornasse pois ele estaria na Fisk University no outono; ela então postergou sua visita à Bahia até ter a chance de inteirar-se sobre as pesquisas de Mr. Pierson. Ela passou os últimos seis meses em Fisk, planejando partir em viagem ao Rio no dia 23 de abril.]. Mais adiante, na mesma carta, escreve: She will probably have letters from Mr. Pierson to Arthur Ramos and some of the other local sarvants [Ela provavelmente terá cartas de Mr. Pierson para Arthur Ramos e alguns outros intelectuais locais]. (Fonte: carta de Robert Park a Hugh Tucker, datada de 5 de abril de 1938. Archival and Manuscript Collections of the Fisk University Franklin Library). tados Unidos em 1947, quando Park já havia falecido, e republicado recentemente no Brasil (2002) com prefácio de Mariza Corrêa e apresentação de Peter Fry. Antiga aluna de Ruth Benedict e de Franz Boas, Ruth Landes dirigiu-se a Robert Park quando pensou em vir à Bahia e, graças a ele, passou, antes de vir ao Brasil, seis meses na Universidade de Fisk, onde se encontrou e discutiu com Donald Pierson. ${ }^{45}$ Robert Park fez-lhe cartas de recomendação a pessoas de sua confiança no Brasil, como Hugh Tucker ${ }^{46}$ e Lois Williams. ${ }^{47}$ Foi a Robert Park que Ruth Landes escreveu várias vezes da Bahia, pedindo-lhe sugestões sobre artigos que havia traduzido (de Edison Carneiro) ou que tinha a intenção de publicar em revistas americanas. ${ }^{48}$ Robert Park, sem

${ }^{47}$ Lois William trabalhava no Instituto Brasil-Estados Unidos. Ela tinha estudado nos Estados Unidos e foi um dos principais contatos do Robert Park no Rio de Janeiro. Diz o Robert Park em carta a Lois William, datada de 5 de abril de 1938: I trust that I am not imposing to great a burden upon you in asking you to give her assistance during her stay in Rio de Janeiro. [...] I am sure you can be of great service to her in making her first contact with Brazilian life and Brazilian people, and you can save her from some of the pitfals that an unwary investigator is likely to fall into. [Eu espero não estar lhe impondo uma carga extra, pedindo-lhe que dê assistência a ela durante sua estadia no Rio de Janeiro [...] Eu estou certo que você pode lhe ser de grande valia para fazer os primeiros contatos com a vida brasileira e com o povo brasileiro, e você pode salvá-la de algumas das ciladas que um investigador incauto provavelmente cairia.] (Archival and Manuscript Collections of the Fisk University Franklin Library).

${ }^{48}$ Escreve Robert Park, em carta datada de 28 de setembro de 1939 a Ruth Landes, após sua volta da Bahia: I was very glad to learn that you are back in this country and I am looking forward to seeing you and hearing from your own lips the story of your adventures in Brazil.[Fiquei muito feliz em saber que você está de volta a esse país e espero ver você e ouvir de sua própria boca a história de suas aventuras no Brasil.]. E continua: I was planning to submit your article to the American Journal of Sociology for publication and I wrote them about the article which you translated from Edison Carneiro. I thought also that you might find an appropriate place in the journal África. I wrote Mr. Westermann a year or more ago telling him of the very interesting remains of African culture in Bahia and that I thought you might want to publish your article and that it was altogether the best thing I have seen. But I understand you have already found a place for it in some other publication. [Eu estava planejando submeter seu artigo à Revista Americana de Sociologia para publicação e lhes escrevi sobre o artigo que você traduziu de Edison Carneiro. Eu também pensei que você pudesse encontrar um lugar apropriado na revista África. Eu escrevi a Mr. Westermann um ano ou mais atrás falando-lhe dos vários vestígios interessantes da cultura africana na Bahia, dizendo-lhe que você pretendia publicar um artigo e que de modo geral sua produção é a melhor coisa que eu já tinha visto. Mas eu a compreendo se você já encontrou um lugar para ele em alguma outra publicação.] (Archival and Manuscript Collections of the Fisk University Franklin Library). 
dúvida, desempenhou um papel importante na sua estadia em Salvador, pois lhe abriu as portas no Brasil, assegurando-lhe ser a Bahia um terreno fértil para seus estudos sobre candomblé. ${ }^{49}$

Depois da Ruth Landes, foi a vez de Melville Herskovits, antropólogo famoso que também passou vários meses em Salvador fazendo pesquisas e que manteve uma longa correspondência com Arthur Ramos (Guimarães, 2004b). Segundo Chor Maio (1997), Herskovits veio ao Brasil e à Bahia pela primeira vez em 1941, muito embora tenha enviado trabalhos ao $1^{\circ} \mathrm{e}$ ao $2^{\circ}$ Congressos Afro-Brasileiros aos quais não pôde comparecer. Esteve na Bahia após ter estado no Daomé, no Haiti, em Trinidad e Suriname. Sua relação com Robert Park é meio ambígua, pois, apesar de ambos se interessarem por estudos comparativos e pelas relações raciais, tinham posições diferentes quanto ao papel da cultura africana no processo de aculturação no "Novo Mundo". ${ }^{50}$ Enquanto Herskovits defendia que diversos aspectos dos traços da cultura africana permaneceram no processo de aculturação (como a possessão), Park estava mais interessado no processo de assimilação $0^{51} \mathrm{e}$ na mobilidade social dos negros. Não por acaso Pierson viu, na Bahia, uma organização social que assumia a forma de uma ordem de livre competição, na qual os indivíduos encontram seu lugar pelos critérios da competência e realizações pessoais e circunstâncias fortuitas, mais que por sua origem racial (Pierson, 1971, p. 365). Pierson não se limitou a estudar as marcas do africanismo na cultura brasileira - as heranças africanas-mas considerou, sobretudo, a miscigenação

${ }^{49}$ Diz a própria Ruth Landes em carta ao Park datada de 30 de Setembro de 1939: Won't you write? After all a man of your position carries the obligation of being permanently inspiring to younger workers in reated fields. I already owe so much to you (....)Affectionately, Ruth Landes.[Porque você não escreve? Afinal, todo homem de sua posição carrega a obrigação de estar permanentemente inspirando os mais jovens que trabalham no mesmo campo de ação. Eu já devo tanto a você.... Afetuosamente, Ruth Landes.] (Archival and Manuscript Collections of the Fisk University Franklin Library).

${ }^{50}$ Park fez uma apreciação sobre um dos livros de Herskovits no American Journal of Sociology. Ver Referências.

${ }^{51}$ A teoria do ciclo das relações raciais desenvolvida por Park dizia que a integração respeitava um padrão sequencial. De início, haveria um processo de competição, seguido pelo conflito, desdobrando-se na acomodação, até finalmente chegar à assimilação. e os novos canais de ascensão social, apostando na hipótese assimilacionista à qual Park se filiava.

A Bahia, nos anos 1940, tornara-se, com efeito, um terreno privilegiado para pesquisadores estrangeiros.

Não cessaram, a partir de então, as vindas de vários deles, inclusive Franklin Frazier, sociólogo e ex-aluno de Park em Chicago, que aqui esteve em 1941, na mesma época em que Herskovits passava seus seis meses em Salvador. Seguindo os passos de Pierson, "Frazier acreditava que o Brasil era um exemplo singular de uma sociedade multirracial de classes com reduzida taxa de tensões étnicas se comparado com a experiência norteamericana" (Maio, 1997, p.183).Éconhecida a controvérsia entre Herskovits e Frazier, que chegou a gerar artigos polêmicos na American Sociological Review, ${ }^{52}$ tendo como pano de fundo a realidade baiana. Para Frazier, que entrevistara famílias negras, a nova dinâmica urbano-industrial fez com que a tradição do candomblé viesse a se transformar em folclore, sendo "as práticas culturais baianas", na verdade, parte da cultura nacional brasileira.

As polêmicas ajudaram, sem dúvida, a Bahia a se tornar um campo favorável às pesquisas. Os estudos sobre contato racial e sincretismo se multiplicavam. Lá fora, ouvia-se falar da Bahia com suas tradições, seu sincretismo religioso, seus terreiros e suas mães de santo... Roger Bastide, por exemplo, antropólogo francês que residia em São Paulo, fascinou-se pela descoberta da África em território brasileiro, estudando as religiões negras, o transe e a possessão do ritual do candomblé (Bastide, 1958).

No final dos anos 1940, sociólogos da Universidade de Columbia juntaram-se com antropólogos e sociólogos brasileiros (Wagley, Charles; Azevedo, Thales de; Costa Pinto, Luiz, 1950) e desenvolveram, no estado da Bahia, o Projeto UNESCO. Originalmente sob a direção de Arthur Ramos (que faleceu quando o projeto estava prestes a iniciar-se), o estudo se voltava para as questões levantadas pela convivência de raças na for-

${ }^{52}$ Ver, nas referências, os artigos de Frazier e de Herskovits. 
mação e história do país. O Projeto se desenvolveu também em outros contextos brasileiros (em São Paulo, no Rio de Janeiro e em Pernambuco). Segundo Chor Maio (1997, p. 50), o deslocamento do campo de pesquisa da Bahia (em princípio, único lugar a ser investigado) para outros estados brasileiros indica a preocupação de se atentar menos para a política de combate ao racismo e mais para as possibilidades abertas pelas ciências sociais, que já começavam seu processo de institucionalização. OBrasil, visto por meio da Bahia, apresentaria o país ao mundo mediante relações raciais harmoniosas. Como contraponto, o Sudeste do Brasil se fazia necessário: na região mais urbanizada e industrializada, as tensões raciais se faziam presentes.

Não é o caso aqui de listar todos aqueles que, desde então (anos 1960 em diante), têm se mobilizado no campo de estudos de relações raciais no Brasil, hoje representados pelos estudos de identidade racial e racismo (Guimarães, 2004a). Preconceito de cor, preconceito de raça, preconceito de marca, desigualdades raciais, discriminação racial e racismo são noções presentes na atual sociologia das relações raciais, campo renovado e retomado nas ciências sociais brasileiras.

No âmbito da política, desde 2002 vem se implantando o sistema de cotas em várias universidades, visando a diminuir a desigualdade racial. A Universidade do Estado da Bahia (UNEB) foi uma das primeiras, reservando $40 \%$ das suas vagas de vestibular aos agora chamados "afrodescendentes" (pretos e pardos). De certo modo, a Bahia faz jus a sua população mestiça, tão presente nos vários estudos e análises que transformaram a cidade do Salvador em um laboratório social.

\section{À GUISA DE CONCLUSÃO}

Howard Becker, numa das vezes em que esteve no Brasil, me disse: one paper, one idea [uma ideia por artigo]. Qual é, portanto, o meu "ponto" neste artigo?

Eu quis mostrar, em primeiro lugar, que, na historia das ciências sociais no Brasil, a visita do
Robert Park a Salvador, na Bahia, muito embora pouquíssimo conhecida, foi importante. Apesar de curta (um mês e meio) foi intensa e ocorreu quando Donald Pierson já tinha concluído seu trabalho de campo e estava prestes a deixar Salvador. Como já mencionado anteriormente, Pierson foi de Salvador para Nashville, onde morou na casa do Park e redigiu sua tese. Orientando e orientador tiveram, portanto, um contato muito próximo, o que permite inferir que as ideias de Park estavam muito presentes no texto escrito por Pierson. Após defender sua tese, Pierson voltou ao Brasil, a São Paulo, onde, nos seus dezesseis anos na Escola de Sociologia e Política, tentou replicar a "Escola de Chicago" da época do Robert Park. Após a morte do mestre (em 1944), continuou difundindo suas ideias e sua prática de pesquisa no Brasil.

E que impacto no próprio Park teve sua visita a Salvador? Tudo nos conduz a concluir que essa visita lhe deixou marcas e indagações. Não que se tenha fascinado pelo ritual do candomblé, como soe acontecer com inúmeros estrangeiros. O grande impacto deve ter sido o de constatar uma realidade diferente da americana, fato que suscitou provavelmente inúmeras questões e interpretações teóricas, que, de imediato, não pôde "resolver".

Eu pretendi mostrar também que Salvador foi transformada num laboratório social a partir da chegada de Donald Pierson, o que foi reforçado com a visita de seu orientador. Os pesquisadores americanos que vieram logo depois se dirigiram à cidade já seguros e legitimados: estavam diante de uma "realidade" que valia a pena ser desvendada, estudada, conhecida, pesquisada com afinco. Donald Pierson havia começado uma discussão que se prolongaria num terreno fértil... A construção social da Bahia (Salvador) como laboratório estava dada...

Uma questão fica em suspenso: e os pesquisadores vindos de outros países que não os Estados Unidos eram portadores de outras questões? A Bahia também se tornou um laboratório social para brasileiros como Edison Carneiro, Thales de Azevedo e tantos outros que até hoje se debruçam sobre a questão do contacto e das desi- 
gualdades raciais? Estas, sem dúvida, são indagações que permanecem.

(Recebido para publicação em março de 2010) (Aceito em abril de 2010)

\section{REFERÊNCIAS}

ANDERSON, Nels. The Hobo. The sociology of the homeless man. Chicago: The University of Chicago Press, 1923.

AZEVEDO, Thales de. As elites de cor: um estudo de ascensão social. São Paulo: Cia. Editora Nacional, 1955.

BASTIDE, Roger. Le candomblé de Bahia (rite Nagô). Paris le Haye: Mouton et Cie., 1958.

CAHNMAN, Werner J. Robert E. Park at Fisk. Journal of the History of the Behavioral Sciences; v. 15, n.4, Out, p. 328-336, 1978.

CARNEIRO, Edison. Candomblés da Bahia. Bahia: Secretaria de Educação e Saúde. Publicações do Museu do Estado, n. 8, 1948.

CHAPOULIE, Jean-Michel. La tradition sociologique de Chicago 1892-1961. Paris: Editions du Seuil, 2001.

CORRÊEA, Mariza. História da Antropologia no Brasil (1930-1960). Testemunhos: Emílio Willems e Donald Pierson. São Paulo: Editora Revista dos Tribunais; Campinas: Editora da Unicamp/Edt. Vértice, 1987.

COSER, Lewis. Masters of sociological thought. New York: Harcout Brace Johanovich. 1971.

COULON, Alain. L'École de Chicago. Paris: PUF, Coll. Que Sais-je? 1992. (Tradução em português: Papirus Editora, 1995).

CUNHA, Olívia Maria Gomes da. Tempo imperfeito: uma etnografia do arquivo. Mana. v. 10, n. 2, Out., p. 287-322, 2004 .

DENIS, Marie-Noele. Robert E. Park à Strasbourg. In: GUTH, Suzie, (Org.). Modernité de Robert Ezra Park. Les concepts de l'École de Chicago. Paris: L' Harmattan, 2008. p.71-81

DURHAM, Eunice; CARDOSO, Ruth. O ensino da antropologia no Brasil. Revista Antropológica da USP, São Paulo, v. 9, n. 1-2, jul-dez, p. 91-107, 1961.

FRAZIER, Franklin, The negro family in Bahia, Brazil. American Sociological Review, n. 7, p.465-478, 1942.

FREYRE, Gilberto. Social Life in Brazil in the middle of the XIX century. Hispanic American Historical Review, v. $5, n^{\circ} 4$, nov., 1922 .

\section{Oficial,1933.}

Casa-Grande e Senzala, 2 vls., Recife: Imprensa

Nordeste, aspectos da influencia da canna sobre $\bar{a}$ vida e a paisagem do nordeste do Brasil. Rio de Janeiro: Livraria José Olympio Editora,1937.

GUIMARÃES, Antonio Sérgio A., Preconceito de cor e racismo no Brasil. Revista de Antropologia, v. 47, n. 1, p. 9-44, 2004a.

Comentários à correspondência entre Melville Herskovits, e Arthur Ramos (1935-1941). In: PEIXOTO, Fernanda Áreas; PONTES, Heloisa e SCHWARCZ, Lilia M. Antropologia, história, experiências. Belo Horizonte: Editora UFMG, 2004b. p. 169-198.
GRAFMEYER, Yves; JOSEPH, Isaac. (Orgs.). L 'École de Chicago. Naissance de l'ecologie urbaine. Paris: Champ Urbain, 1979.

GROSSMAN, James R.; KEATING, Ann Durkin e REIFF, Janice L. (Edts.). The encyclopedia of Chicago. Chicago: the University of Chicago Press, 2004.

GUTH, Suzie (Org.) Modernité de Robert Ezra Park. Les concepts de l'Ecole de Chicago. Paris: L' Harmattan, 2008.

Masse und Publikum. La thèse de Robert Park. In: GUTH, Suzie, (Org.). Modernité de Robert Ezra Park. Les concepts de l'École de Chicago. Paris: L' Harmattan, 2008. p. 31-58.

HALBWACS, Maurice [1932]. Chicago experience ethnique. In: GRAFMEYER, Yves e JOSEPH, Isaac. (Orgs.) L'École de Chicago. Naissance de l'écologie urbaine. $1^{\mathrm{a}}$ edição [1979]. Paris, Champs/Flammarion, 2004.

HERSKOVITS, Melville. The negro in Bahia, Brazil: a problem in method. American Sociological Review, n. 8, p. 394-402, 1943.

HULL-HOUSE MAPS AND PAPERS. A presentation of nationalities and wages in a congested district of Chicago, together with comments and essays on problems growing out of the social conditions by the residents of Hull-House. [1 $1^{\mathrm{a}}$ ed., 1895]. Urbana, Chicago: University of Illinois Press, Introdução de Rima Lunin Schultz, 2007.

IBGE. Instituto Brasileiro de Geografia e Estatística, Enciclopédia dos Municípios Brasileiros, Rio de Janeiro: IBGE, v. XXI, 1958.

JOHNSON, Charles. The negro in Chicago. A study of race relations and a race riot in 1919. Chicago: the University of Chicago Press, 1922.

Robert E. Park: in memoriam. Sociology and Social Research, v. 28, (1943-44), Phylon, v. VI, 1945.

LANDES, Ruth, [1947] A Cidade das Mulheres. Rio de Janeiro: Editora UFRJ, 2002.

LECLERC, Gérard. L'observation de l'homme. Une histoire des enquêtes sociales. Paris: Le Seuil, 1979.

LEVINE, Robert M. The first Afro-Brazilian Congress: opportunities for the study of race in the Brazilian Northeast. Race, v. XV, n. 2, p. 185-193, 1973.

LIMONGI, Fernando. A Escola Livre de Sociologia e Política em São Paulo. In: MICELI, Sérgio (Org.). História das ciências sociais no Brasil. São Paulo: Vértice; Editora Revista dos Tribunais, IDESP, v. 1, 1989. p. 217-233.

MAIO, Marcos Chor. A história do Projeto UNESCO. Estudos raciais e ciências sociais no Brasil, 1997. Tese [Doutorado em Ciências Políticas] - Programa de Pós-Graduação em Sociologia e Ciências Política do Instituto Universitário de Pesquisas do Rio de Janeiro- IUPERJ.

MASSI, Fernanda. Franceses e norte-americanos nas ciências sociais brasileiras 1930-1960. In: MICELI, Sérgio (Org.). História das ciências sociais no Brasil. São Paulo: Vértice; Editora Revista dos Tribunais, IDESP, v. 1. 1989. p. $410-459$.

MATTEWS, Fred H. Quest for an American Sociology: Robert E. Park and the Chicago School. Montreal e Londres: McGill-Queen's University Press, 1977.

MENDOZA, Edgar S.G. Donald Pierson e a escola sociológica de Chicago no Brasil: os estudos urbanos na cidade de São Paulo (1935-1950). Sociologias. Porto Alegre, ano 7, $\mathrm{n}^{\circ} 14$, jun/dez, p. 440-470, 2005.

PARK, Robert E. Masse und Publikum. Berne: Buchdruckerei Lack \& Grunau, 1904.

. Human Migration and the Marginal Man. American Journal of Sociology, v. 23 n. 6, May, p. 881-893, 1928. 
Review of Life in a Haitian Valley by Melville J. Herskovits. American Journal of Sociology, v. XLIII, n.2, Sept., p. 346-348, 1937.

The city as a social laboratory. In: SMITH, T.V. WHYTE, Leonard (Orgs.). Chicago: an experiment in social science research. Chicago: The University of Chicago Press, p. 1-19, 1929

Race and Culture. Essays in the sociology of contemporary man. London: The Free Press of Glencoe, 1950.

. The career of the Africans in Brazil. "Introduction" to PIERSON, Donald. Negroes in Brazil. Chicago University of Chicago Press, 1942.

; BURGESS, Ernest. (Orgs.) Introduction to the Science of Sociology (com novo prefácio por Morris Janovitz). $1^{\text {a }}$ edição [1921]. Chicago e Londres: The University of Chicago Press, 1970.

; MCKENZIE, R. The City. Suggestions for the Investigation of Human Nature in the Urban Environment. Chicago: University of Chicago Press, 1925.

PEIXOTO, Fernanda Áreas. Diálogos brasileiros: uma análise da obra de Roger Bastide. São Paulo: Editora da Universidade de São Paulo, 2000.

PIERSON, Donald. Negroes in Brazil: a study of race contac at Bahia. Chicago: The University of Chicago Press, 1942

[1945] Brancos e pretos na Bahia: estudo de contacto racial. Introducão de Arthur Ramos e Robert E. Park. $2^{\mathrm{a}}$ Ed. São Paulo: Editora nacional, 1971.

RAMOS, Artur. [1943] Introdução à Primeira Edição Brasileira. In: PIERSON, Donald. Brancos e prêtos na Bahia estudo de contacto racial. São Paulo: Editora Nacional, 1971. p. 67-70.

RAUSHENBUSH, Winifred. Robert E. Park: biography of a sociologist. Chapel Hill: Univesity of North Carolina Press, 1979.

RODRIGUES, Raimundo Nina. Os africanos no Brasil. São Paulo: Cia Editora Nacional,1935.

ROMO, Anadelia. Rething race and culture in Brazil's First Afro-Brazilian Congresso of 1934. Journal of Latin American Studies, n³9, Fev., p. 31- 54, 2007.
SCHRECKER, Cherry. Robert E. Park et Ernest Burgess; Introduction to the Science of Sociology. In: GUTH, Suzie. (Org.). Modernité de Robert Ezra Park. Les concepts de l'Ecole de Chicago. Paris: L' Harmattan, 2008. p. $213-232$

SHAW, Clifford. The Jackrolle. A delinquent boy's own story. Chicago: the University of Chicago Press, 1930.

SIMMEL, Georg [1908] Digressions sur l'étranger. In: GRAFMEYER, Yves et JOSEPH, Isaac (Orgs.). L'Ecole de Chicago. Naissance de l'écologie urbaine. Paris: Champ Urbain, 1979. p. 53-59.

STONEQUIST, Everett. The marginal man. New York: Charles Scribner's Son, 1937.

TOPALOV, Christian. La ville, lieu de l'assimilation sociale?. In: JAISSON, Marie e BAUDELOT, Christian (Orgs.). Maurice Halbwachs, sociologue retrouvé. Paris: Editions Rue d'Ulm, 2007.p. 87-101.

TUNA, Gustavo Henrique. Primeiros passos em retrospectiva. In: FREYRE, Gilberto, Vida Social no Brasil nos meados do século XIX. 4a. edição revista. São Paulo: Global, 2008.

THRASHER, Frederick. The Gang. A study of 1313 gangs in Chicago. Chicago: the University of Chicago Press, 1927.

VALLADARES, Lícia. (Org.) A escola de Chicago. Impacto de uma tradição no Brasil e na França. Rio de Janeiro: IUPERJ; Belo Horizonte: Editora UFMG, 2005.

VILA NOVA, Sebastião. Donald Pierson e a Escola de Chicago na Sociologia Brasileira. Entre humanistas e messiânicos. Lisboa: VEGA, Gabinete de Edições, 1998.

WAGLEY, Charles; AZEVEDO, Thales, COSTA PINTO, Luiz. Uma pesquisa sobre a vida social no Estado da Bahia. Salvador: Publicacões do Museu do Estado, Secretaria de Educação e Saúde, 1950.

WASHIGTON, Booker T.; PARK, Robert E. [1912], The man farthest down. New Brunswick: Transaction Book, 1984.

WIRTH, Louis. The ghetto. Chicago: the University of Chicago Press, 1929.

ZORBAUGH, Harvey. The gold coast and the slum. Chicago: the University of Chicago Press, 1929. 


\section{THE VISIT OF ROBERT PARK TO BRAZIL, THE "MARGINAL MAN" AND BAHIA AS A LABORATORY}

\section{Licia do Prado Valladares}

This paper is part of the theoretical concerns of the author on the reception of the "Chicago School" in Brazil. It analyzes the passage of Robert Park in the late'30s in Bahia, his motivations and the effects on international social sciences. This trip is little known, and the paper offers a little collaboration to the history of social sciences in Brazil and Bahia. Based on data from original research conducted in Brazil and the United States, the author considers the importance of the visit by Park [and Pierson] to Bahia. It incorporates classical notions of Marginal Man, developed by Park and melting pot, used by Park and disciples, which referred to the conviviality of Chicago communities from different nationalities, who did not mix, distinguishing it from the Bahian case, of miscegenation. This singularity transformed Bahia into a "social laboratory", prompting the arrival of other anthropologists, and new questions and theoretical interpretations, now resumed.

Keywords: Chicago School, Robert Park, Donald Pierson, race relations, Bahia and Brazil.

\section{LA VISITE DE ROBERT PARK AU BRÉSIL, "L'HOMME MARGINAL” ET BAHIA SERVENT DE LABORATOIRE}

\section{Lícia do Prado Valladares}

Cet article fait partie des préoccupations théoriques de l'auteure concernant la venue de "l'école de Chicago" au Brésil. Elle y fait l'analyse du passage de Robert Park à Bahia, à la fin des années 30, de ses motivations et des effets qu'il a eu pour les sciences sociales internationales. Ce voyage est peu connu, ce qui permet à l'article d'apporter une collaboration à l'histoire des sciences sociales au Brésil et à Bahia. Sur la base des données originales d'une recherche menée au Brésil et aux Etats-Unis, l'auteure considère l'importance de la visite de Park [et de Pierson] à Bahia. Elle reprend les notions classiques de l'Homme Marginal, développées par Park, et celles de melting-pot, utilisées par Park et ses disciples, lorsque ce dernier faisait référence à la vie, à Chicago, de communautés de nationalités différentes qui ne se mélangeaient pas. Le cas du métissage bahianais a intrigué Park au point de transformer Bahia en un "laboratoire social" qui a suscité non seulement la venue d'autres anthropologues mais aussi de nouvelles questions et d'interprétations théoriques qui sont reprises actuellement.

Mots-CLÉs: Ecole de Chicago, Robert Park, Donald Pierson, relations raciales, Bahia, Brésil.

Lícia do Prado Valladares - Doutora em Sociologia pela Universidade de Toulouse (Fr.). Livre-docente pela Université de Lyon II. Professora na Universidade de Lille I (França) e membro do CLERSÉ. Foi Professora Titular do IUPERJ (1980-2000), sendo hoje pesquisadora associada. Coordenou a URBANDATA desde 1989, figurando atualmente como coordenadora emérita. Ministrou cursos em universidades estrangeiras como professora convidada (Universidades de Paris; Génève, Budapest e Texas) e foi membro do Comitê do programa MOST da UNESCO. É autora de inúmeros artigos e livros em Sociologia Urbana, sobre favela, pobreza urbana e história da pesquisa urbana, no Brasil, dentre os quais, destacam-se: Passa-se uma casa (Zahar,1978), La favela d'un siècle à l'autre (Paris: Editions da MSH, 2006); A Invenção da Favela (Rio de Janeiro: FGV, 2005) e a coletânea A Escola de Chicago (Belo Horizonte / Rio de Janeiro: Editora da UFMG/ IUPERJ, 2005). 
